\title{
COSMOS, a spatially explicit model to simulate the epidemiology of Cosmopolites sordidus in banana fields
}

Fabrice Vinatier ${ }^{1}$, Philippe Tixier $^{1}$, Christophe Le Page ${ }^{2,}$ Claude Bruchou ${ }^{3}$ \& Françoise Lescourret ${ }^{4}$

${ }^{1}$ CIRAD, UPR 26, B.P. 214, F-97285, Le Lamentin, Martinique, France

fabrice.vinatier@cirad.fr

${ }^{2}$ CIRAD, UPR GREEN, 254 Phayathai road, Pathumwan, 10330, Bangkok, Thailand

${ }^{3}$ INRA, Biométrie, UR 1115, Domaine St. Paul, Site Agroparc, Avignon Cedex 9, 84914, France

${ }^{4}$ INRA, Unité Plantes et Systèmes de Culture Horticoles, UR 1115, Domaine St. Paul, Site Agroparc, Avignon Cedex 9, 84914, France

\begin{abstract}
In French West Indies, cyclones can cause severe damages to banana plants, and banana pests are thus confronted to a lack of resources. Here, we investigate with a spatial model the effect of a catastrophic disturbance, like a cyclone, on the demography and spatial dynamics of a population of banana weevil (Cosmopolites sordidus) in French West Indies. The individual-based model called COSMOS describes the epidemiology of $C$. sordidus in the field. Each biological parameter of the model was estimated from literature and experimental data. The model was validated at the microparcel scale, comparing using the Kolmogorov-Smirnov test observed and simulated distributions of attacks. 12 out of 18 micro-parcels were similar. An exhaustive sensitivity analysis, using the Morris method, allowed evaluating the importance of each parameter in the distribution of attacks. According to model simulations at the field scale, the time necessary for an entire collapse of populations following a shortage of resources is approximately one year, depending only on the mortality rate of adults. Spatial clustering of populations emerges from the model, because of successive grow-up and decline of population associated with limited dispersal capabilities. The model developed here helps us to understand the key factors of the epidemiology of a tropical pest and the dynamics resulting from a major disturbance.
\end{abstract}

Keywords: Individual-based model / Pattern-oriented modelling / Parameter estimation / Population dynamics 